\title{
Ce que sera le Centenaire en Suisse
}

Le programme des manifestations du Centenaire en Suisse s'échelonnera sur une période allant du I5 août au I5 septembre I963. Il doit permettre non seulement de faire le point, au bout de cent ans d'existence, d'une idée qui a fait le tour du monde, mais encore de jeter les bases d'un travail fructueux pour l'avenir, au seuil d'un second siècle. Il sera l'occasion pour les spécialistes de confronter leurs expériences et pour le grand public de s'informer sur la Croix-Rouge et ses activités.

Voici quelles en seront les différentes parties:

I) Un centre international d'étude pour les responsables des services infirmiers de la Croix-Rouge aura lieu à Lausanne, du I9 au 23 août, et réunira des infirmières et aides-infirmières possédant une certaine expérience des activités de la Croix-Rouge. Il offrira l'occasion aux participants de penser à nouveau le rôle du personnel infirmier au sein de la Croix-Rouge, en regard de l'évolution économique et sociale qui se manifeste dans les divers pays.

2) Une rencontre internationale de secouristes de la Croix-Rouge est prévue, du I9 au 23 août, à Macolin près de Bienne. Elle est destinée à des secouristes qualifiés et connaissant bien les méthodes utilisées dans leur Société. Elle se composera d'une partie théorique (exposés techniques, exposés généraux, brefs rapports sur les activités nationales de secourisme) et d'une importante partie pratique comportant notamment des exercices et des démonstrations présentés par les équipes. 
3) Une conférence mondiale d'éducateurs se réunira à Lausanne, du I9 au 23 août. Son but est d'obtenir une coopération accrue du corps enseignant à l'effort entrepris pour répandre l'idée de la Croix-Rouge. Les directeurs des Croix-Rouges nationales de la Jeunesse y participeront, mais aussi les membres du corps enseignant de divers degrés - primaire, secondaire, universitaire et écoles techniques - et par les représentants des ministères nationaux de l'éducation, des médecins des écoles et des représentants des ministères de la Santé publique. Elle étudiera, dans ses différents aspects, le programme de la Croix-Rouge de la Jeunesse, les moyens de le réaliser à l'intérieur des programmes scolaires et de développer une coopération plus étroite entre le corps enseignant et la Croix-Rouge de la Jeunesse. Un autre sujet proposé est l'adaptation de la Croix-Rouge de la Jeunesse aux tendances du monde moderne.

4) Un séminaire sur l'activité de la Croix-Rouge en cas de conflits armés, de troubles intérieurs ou de tension internationale, ouvert à tous les dirigeants ou membres de la Croix-Rouge que ces problèmes intéressent, sera organisé à Genève du I9 au 23 août. On y passera en revue les différents aspects de l'activité que les institutions de la Croix-Rouge, tant nationales qu'internationales, peuvent exercer dans ces circonstances en faveur des victimes. Il traitera aussi de la diffusion des Conventions de Genève et des moyens d'intensifier et d'améliorer encore l'action de la Croix-Rouge dans ce domaine.

5) Les 24 et 25 août, des manifestations sont prévues dans la région de Colombier (Neuchâtel) à l'intention des participants aux diverses rencontres et réunions énumérées ci-dessus, qui se réuniront autour d'un feu de camp et assisteront à des démonstrations organisées en collaboration avec le Service de santé de l'armée suisse et la Croix-Rouge suisse.

6) Durant la semaine du 25 au 3I août, en guise de préliminaires aux réunions des institutions internationales de la Croix-Rouge, on prévoit d'organiser, à Genève, des manifestations d'ordre général ouvertes à tous. Les membres de la Croix-Rouge pourront ainsi, après avoir participé, la semaine précédente, à des réunions tech- 
niques séparées, se retrouver et se mêler au grand public. Voici ce que seront ces manifestations:

a) Le matin, des conférences-entretiens auront pour thème " $\mathrm{La}$ Croix-Rouge dans le monde d'aujourd'hui ". Des exposés généraux seront suivis d'échanges de vues, qui porteront sur des sujets tels que "L'évolution des tâches de la Croix-Rouge: possibilités et limites " ou "La Croix-Rouge, facteur de rapprochement entre les peuples". Tous pourront de cette manière se familiariser avec les grands problèmes qu'affronte la CroixRouge, au seuil de son. deuxième siècle d'existence.

b) L'après-midi, on prévoit des démonstrations publiques de secourisme, de soins infirmiers, de transfusion de sang et des visites que les membres de la Croix-Rouge présents à Genève voudraient rendre au CICR et à la Ligue.

7) Une journée commémorative aura lieu le $\mathrm{I}^{\mathrm{er}}$ septembre, dont le programme a été exposé ici-même ${ }^{\mathbf{1}}$. Les juniors y auront une large place et on envisage à cette occasion de monter un ballet et un spectacle folklorique.

8) Un Congrès du Centenaire de la Croix-Rouge internationale se réunira à Genève, du 27 août au Io septembre, en lieu et place de la $\mathrm{XX}^{\mathrm{e}}$ Conférence internationale de la Croix-Rouge, qui a été ajournée de deux ans. Seuls les représentants de la Croix-Rouge auront le droit de participer aux deux sessions dont la première, qui durera du 27 au $3 \mathrm{r}$ août, sera la $27^{\mathbf{e}}$ session du Conseil des Gouverneurs de la Ligue des Sociétés de la Croix-Rouge. La seconde, celle du Conseil des Délégués de la Croix-Rouge internationale, composée des représentants de plus de go Sociétés nationales de la Croix-Rouge, du Croissant-Rouge et du Lion-et-Soleil Rouge, du Comité international et de la Ligue, siégera du 2 au ro septembre. Le Conseil des Délégués traitera les questions prévues à l'ordre du jour de la $\mathrm{XX}^{\mathrm{e}}$ Conférence internationale, à l'exception de celles statutairement réservées à cet organe.

1 Voir Revue internationale, novembre 1962. 
9) Des démonstrations seront organisées, les 6 et 7 septembre dans les régions alpines, par le Service de santé de l'armée suisse et la Croix-Rouge suisse.

ro) Enfin, pendant toute la période des manifestations, soit du I5 août au I5 septembre, sera ouverte au public l'Exposition internationale de la Croix-Rouge, dont le programme a déjà été esquissé ici-même ${ }^{1}$, mais dont nous voudrions souligner l'importance en précisant sa conception générale.

Conçue en fonction des diverses catégories de visiteurs qui la parcourront, l'Exposition doit constituer un excellent moyen d'information pour faire comprendre ce qu'est la Croix-Rouge, quels sont ses buts et ses principes. Tel est son objectif essentiel et elle sera donc ouverte le plus largement possible, en particulier aux jeunes qui seront amenés, dans l'avenir, à prendre la relève.

On voudrait obtenir un effet durable, de façon que le visiteur, après s'être rendu compte de ce que la Croix-Rouge peut faire pour lui, se demande à son tour ce qu'il pourrait faire pour elle. Elle s'inscrit donc au nombre des moyens destinés à soutenir la campagne de recrutement qui sera lancée l'année prochaine, justification de l'activité de la Croix-Rouge et de l'appui qu'on lui accorde. Enfin, elle pourrait former l'embryon du futur musée permanent dont le besoin se fait sentir depuis longtemps.

Une fois précisé le dessein de l'entreprise, il s'agissait de trouver les moyens de le réaliser, en se souvenant que l'idéal de la CroixRouge implique un niveau et une tenue aussi élevés que possible, et qui restent dans les limites imposées par une stricte économie.

Aussi, simplicité et clarté furent-ils les deux thèmes dont on s'inspira dès le début: simplicité dans le choix des matériaux (recherche constante de la solution la plus simple qui est souvent la plus belle) mais surtout clarté: l'ordre chronologique dès lors s'imposa. Dans la section historique, bien sûr, mais aussi, par exemple, dans le secteur "aide aux victimes de conflits armés",

1 Voir Revue internationale, novembre r962. 


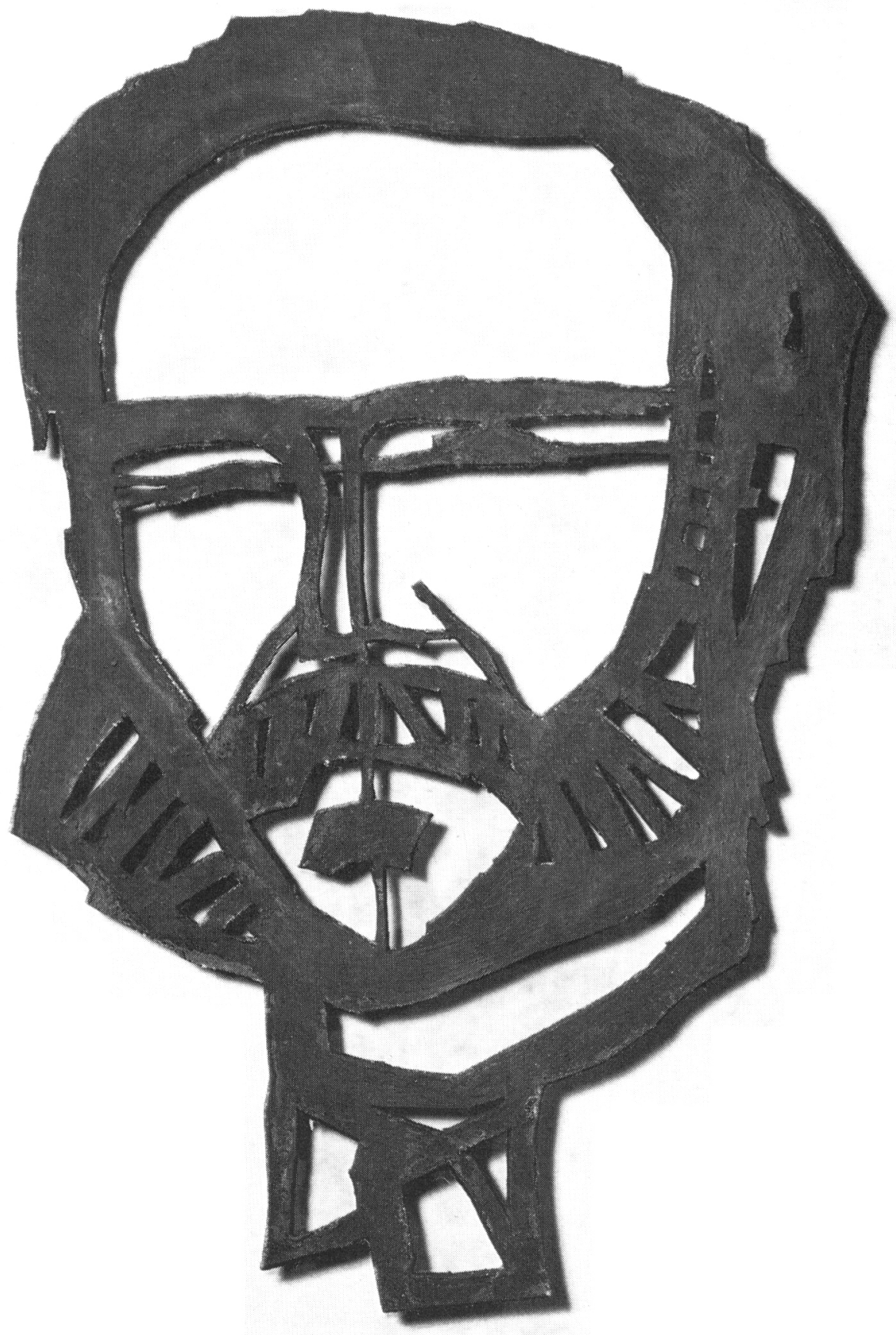

Ce portrait de l'anteur d'Un souvenir de Solférmo ornera le bateau qui sera lancé, durant l'année du Centenaire, sur le lac Léman, et qui portera le nom de Henry Innant. Fuvre du graveur Robert Héritier, il est découpé dans du métal. 


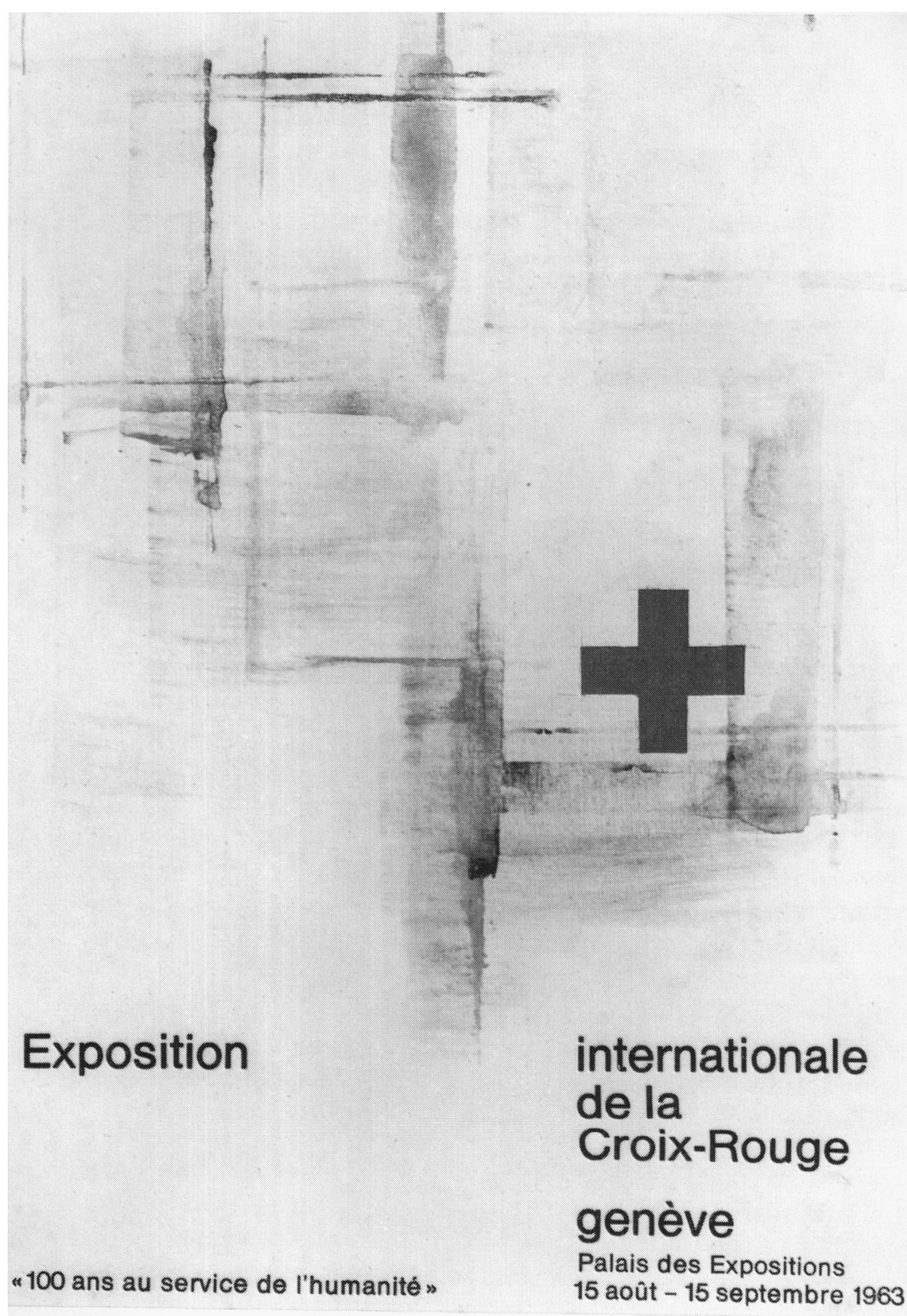

Projet de l'affiche officielle de l'Exposition internationale de la Croix-Rouge.

(Auteur: Michel Gallay) 
puisqu'on se préoccupa d'abord du sort des blessés militaires et des naufragés, puis, plus tard, de celui des prisonniers de guerre et enfin des civils.

Ainsi le visiteur suivra-t-il le développement historique de la Croix-Rouge et se familiarisera-t-il avec ses activités dans l'ordre chronologique de leur naissance. Le sens unique s'impose donc de lui-même, logiquement. Les présentations n'apparaîtront en principe que sur l'un des côtés du chemin à suivre et les parois qui feront face devront, dans la mesure du possible, favoriser le repos de l'œil.

Toujours pour faciliter la compréhension du public, les différents sujets de cette exposition, thématique par excellence, seront présentés sous forme de chapitres formant un tout en eux-mêmes et nettement délimités dans l'espace. De plus, chaque secteur sera placé sous une couleur dominante, dont le changement fera mieux saisir le passage d'un thème à un autre.

Enfin, des "reposoirs" seront installés pour permettre aux visiteurs d'assimiler ce qui leur aura été présenté et de se restaurer, tout en ayant à portée de main une documentation spécialement choisie $^{1}$.

La section historique, première partie de l'exposition, montrera le développement de la Croix-Rouge depuis sa fondation jusqu'à l'actualité immédiate dont il sera possible de prendre connaissance par un tableau tenu continuellement à jour. A l'entrée déjà, le visiteur sera saisi et plongé dans l'atmosphère qui a suscité la naissance de la Croix-Rouge. Les dates les plus marquantes apparaîtront dans leur contexte, qui sera jalonné d'un certain nombre de points de repère (grandes inventions, par exemple). Certains faits seront présentés sous forme de "spectacles", pour en faciliter la compréhension.

La deuxième partie sera consacrée à la section actualité. Elle fera ressortir l'unité et la diversité de la Croix-Rouge, unité à cause de son idéal commun à tous les pays et diversité qui résulte des besoins nationaux différents. La présentation de quelques activités spéci-

1 En hors-texte, on peut voir le projet de l'affiche choisie, à la suite du concours organisé par le Département fédéral de l'Intérieur, en liaison avec la Commission suisse des arts appliqués. 
fiques à certaines Sociétés nationales permettra d'introduire un élément exotique et de créer des attractions. Il en sera ainsi de la réplique du serpentarium de Bangkok, qui contiendra 500 serpents des espèces les plus venimeuses, et où des spécialistes procéderont à la prise de venin pour la préparation de vaccins.

On s'efforcera de montrer que, même après cent ans, la CroixRouge est bien vivante et qu'elle est prête à affronter les nouvelles tâches qui s'offrent continuellement à elle. Après un premier secteur de transition relatif à l'organisation de la Croix-Rouge internationale et le droit humanitaire, les secteurs suivants traiteront des diverses activités de la Croix-Rouge: aide aux victimes de confits armés; assistance aux victimes de catastrophes; santé; Croix-Rouge de la Jeunesse.

Enfin, le secteur conclusions - idée qui, sous la forme envisagée, peut très probablement passer pour une innovation dans la technique d'exposition - présentera, en manière de condensé, les problèmes qui se sont posés à la Croix-Rouge et les solutions qu'elle y a apportées. En outre, le visiteur s'arrêtant à un "reposoir" conçu d'une manière fort originale, comprendra qu'il peut aider - personnellement - la Croix-Rouge à accomplir ses tâches.

Le premier étage sera réservé à l'information - presse écrite, parlée, filmée et télévisée - à la numismatique, à l'affiche Croix-Rouge, ainsi qu'à la philatélie (I35 territoires ont émis des timbres Croix-Rouge et l'on montrera ici la collaboration souvent émouvante des administrations postales et de la CroixRouge). Deux salles de cinéma présenteront, l'une, un programme général à l'intention du grand public et, l'autre, des films d'un intérêt particulier.

Du matériel sanitaire militaire sera exposé dans différents secteurs, et la participation des services de santé militaires permettra de faire ressortir le parallélisme qui existe entre les services de santé et la Croix-Rouge, et de souligner l'importance des Conventions humanitaires.

Enfin, on étudie la création d'une section dite du "matériel mis au service de l'homme " qui offrirait à l'industrie et au commerce la possibilité de présenter celles de leurs réalisations qui peuvent être utilisées par la Croix-Rouge et les services de santé des armées. 
En résumé, les idées, plutôt que d'être imposées, seront suggérées par un agencement logique. Comme l'a justement relevé un expert en la matière, une exposition n'est point un livre. Le public est, en effet, saturé d'expositions à deux dimensions (photos-textes). Une troisième dimension - voire une quatrième - devra absolument être introduite partout où cela sera de rigueur. L'objet que l'on peut, de préférence, toucher, sera à la place d'honneur, la maquette aussi. Des "éléments parlants" et le groupement judicieux des objets présentés permettront de réduire le texte au maximum. Enfin, des dispositifs mobiles, visuels ou acoustiques, solliciteront, dans la mesure du possible, la participation du visiteur. Sans tomber dans le travers de la recherche du nouveau à tout prix, les organisateurs veulent réaliser une exposition qui fasse date. Avant tout, un climat favorable doit être créé. L'unité de conception sera respectée, de façon que cette manifestation constitue un tout homogène. Mais unité ne veut pas dire uniformité et, par une technique de présentation aussi variée que possible, on assurera une heureuse diversité.

On le voit, ce que veulent les membres des Commissions, Sous-Commissions et groupes créés pour élaborer le programme du Centenaire en Suisse, de même que les commissaires et graphistes qui ont la charge de penser et d'organiser l'exposition, c'est mettre en lumière l'idée de la Croix-Rouge et illustrer un des thèmes retenus pour le Congrès du Centenaire de la CroixRouge: "Cent ans au service de l'humanité. " Nous souhaitons que nombreux soient ceux qui, venus du monde entier pour assister aux manifestations prévues et visiter l'exposition, se rendront compte que la Croix-Rouge, partout, est vivante et efficace.

\section{EDOUARD-J. LOGOZ}

Secrétaire général de la Commission du Centenaire de la Croix-Rouge en Suisse et commissaire général de l'Exposition 